\title{
APPLICATION OF SELECTED MOMENTUM CORRECTION METHOD USING INDUCTION VOLTAGE MODULATOR
}

\author{
T. Kikuchi*, S. Kawata, Utsunomiya University, Utsunomiya, 321-8585, Japan \\ T. Katayama, CNS, University of Tokyo, Saitama, 351-0198, Japan \\ K. Horioka, Tokyo Institute of Technology, Yokohama, 226-8502, Japan
}

\begin{abstract}
A method for momentum correction of a selected beam particle using a controllable induction voltage modulator is proposed for a low flux ion beam. The corrected ion beam has a small momentum error restricted by a detection error at a kinetic energy analyzer and a voltage fluctuation at the induction voltage modulator. Numerical simulation indicates that the scheme using a multi-turn correction in a storage ring can considerably reduce the requirement of the amplitude and the tolerance level of the correction voltage.
\end{abstract}

\section{INTRODUCTION}

Radioactive isotope (RI) beam is important for developing nuclear physics studies on reactions and properties of unstable nuclei [1]. At GSI and RIKEN facilities, such mass spectroscopy experiments using rare and unstable RI beams are planned in the near future [2, 3, 4]. RI beams produced by projectile fragmentation in heavy ion collision are collected by RI beam separators [5]. It is predicted that typical production rates of the secondary beams are low as 1 to $1000 \mathrm{~s}^{-1}$, so that the RI beam must be efficiently utilized for experiments. The quality of RI beam is the most important parameter for the injection into the storage ring and for the guidance to the experimental apparatus. Such RI beam separator has a rather large acceptance in the transverse plane and momentum spread for the rare RI collection. Since the storage ring and the experimental setup have a finite acceptance, the injection and guidance of the RI beam with large transverse emittance and large momentum spread must be regulated. For the improvement of transverse emittance, individual trajectory correction and individual injection schemes were proposed [6]. The parameters of each RI are measured individually, and the particle trajectory can be corrected by a fast kicker system. Although it is expected that the trajectory of beam particle is transversely corrected using the scheme and the RI beam is efficiently injected into the storage ring, the large momentum spread still remains during the transport and injection.

Induction voltage modulator, which is based on a voltage adder configuration, can make arbitrary waveform. The induction voltage module, which has a precise waveform controllability and a high repetition rate, is developed $[7,8]$. Since the operation of induction voltage modulator is based on solid-state power devices, we can apply the

\footnotetext{
*tkikuchi@cc.utsunomiya-u.ac.jp
}

voltage to a charged particle beam in a controllable manner.

We propose a selected momentum correction method of each charged particle using the induction voltage modulator. When the momentum error from the destination energy of the selected particle is detected, the induction voltage modulator can individually kick the particle for the momentum correction. The repeating operation of the method brings the rapid reduction of the momentum error. Using the proposed scheme, the momentum error of each charged particle is corrected, and we will be able to use the cooled beam for the above purposes.

\section{SELECTED MOMENTUM CORRECTION METHOD}

Figure 1 shows the outline of this scheme. The parti-

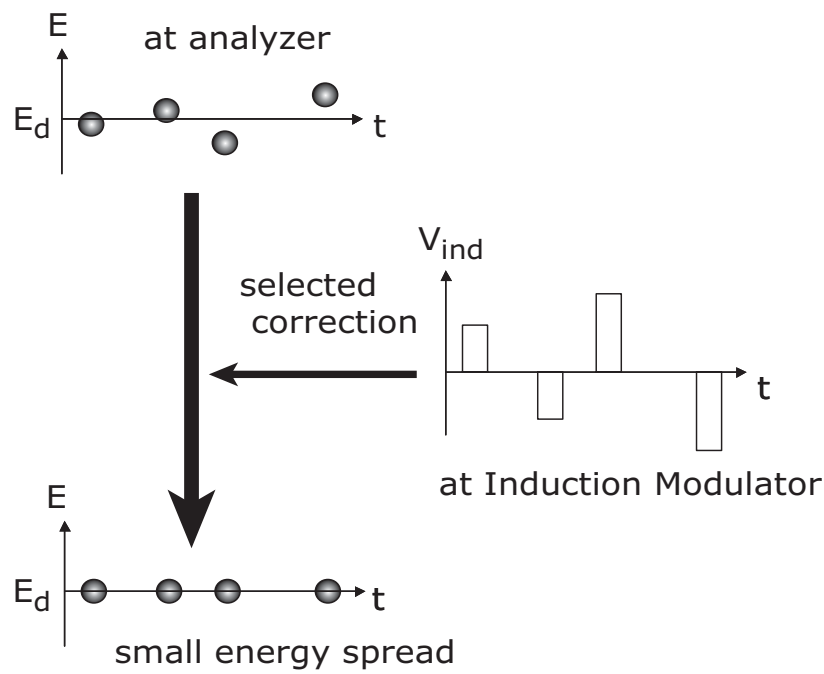

Figure 1: Outline of selected momentum correction scheme.

cle energy can be detected by an analyzer at the entrance of beam transport line. The discrepancy of particle energy from the destination value is given by the information from the analyzer. For compensation of the detected energy difference, the induction modulator can apply the correction voltage. Consequently, the particle energy is corrected one by one to the destination energy, and the momentum error approaches to zero, rapidly. Using the proposed scheme, we can control the longitudinal momentum of the beam particle, efficiently. This selected momentum correction 
scheme together with the individual trajectory correction also provides us a novel method of fast beam cooling.

The voltage applied by induction modulator can regulate each particle energy. The relation between the corrected particle energy $E_{c}$ and the applied voltage $V$ is

$$
E_{c}=E_{i}+q V
$$

where $E_{i}$ is the initial particle energy before the gap of induction unit and $q$ is the charge state of the particle. The applied voltage is assumed as $V=V_{i n d}+\Delta V_{f}$, where $V_{i n d}$ is the voltage from the induction modulator and $\Delta V_{f}$ is the fluctuation of voltage applied by the induction module, respectively.

Using the analyzer based on the time-of-flight (TOF) method [9], we are able to determine the particle energy with a somewhat high accuracy. However the analyzer has an error from the true value of the particle energy. As a result, the analyzed particle energy $E_{a}$ is given as $E_{a}=E_{i}+\Delta E_{a}$, where $\Delta E_{a}$ is the error at the analyzer.

The voltage required at the induction modulator is determined by

$$
q V_{\text {ind }}=E_{d}-E_{a},
$$

where $E_{d}$ is the destination particle energy. Figure 2 shows the relations between the particle energy, the voltage applied by the induction module, and the destination energy, respectively. Here the voltage applied by the induction (a)

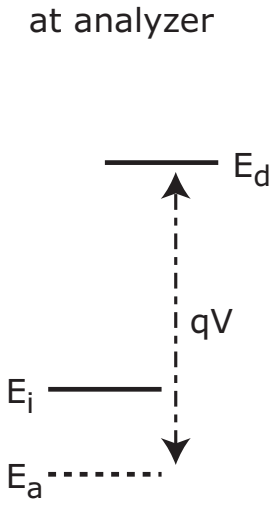

(b)

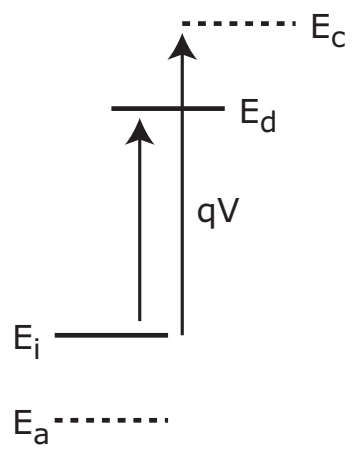

after induction gaps

Figure 2: Particle energy modulated by an induction voltage modulator for $V=V_{\text {ind }}+\Delta V_{f}<V_{\text {limit }}$, (a) at the analyzer and (b) after the induction voltage modulator.

module is assumed as $V=V_{\text {ind }}+\Delta V_{f}<V_{\text {limit }}$, which is the voltage limitation of the induction module. As an ideal situation without any fluctuations and errors, the condition of $E_{d}=E_{c}$ is satisfied, i.e., $q V_{i n d}=E_{d}-E_{i}$. In this case, the modulated particle energy is replaced by $E_{c}=E_{i}+q\left(V_{\text {ind }}+\Delta V_{f}\right)$, for $\left|E_{d}-E_{a}\right| \gg q V_{\text {limit }}$. Figure 3 shows the estimated voltage required to the induction module for the momentum correction.

Substituting Eq. (2) to Eq. (1), the modulated particle energy is rewritten by $E_{c}=E_{d}-\Delta E_{a}+q \Delta V_{f}$. From

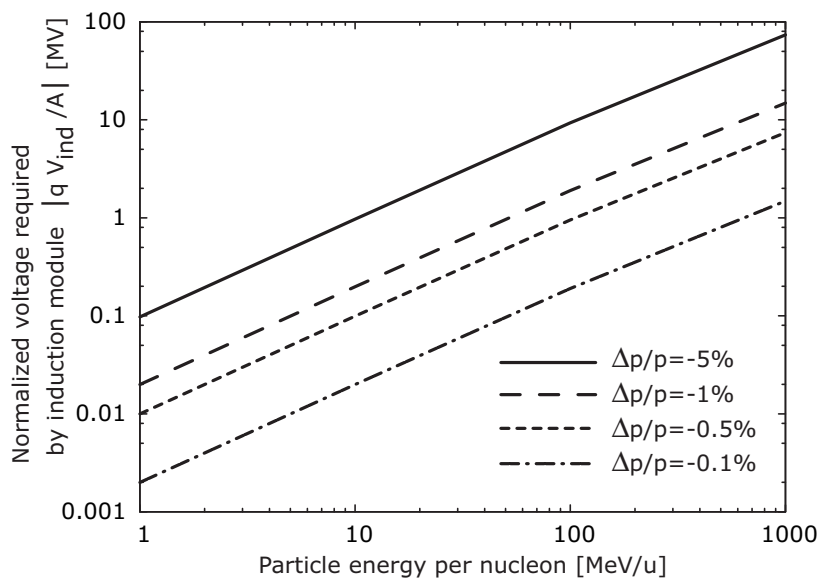

Figure 3: Voltage required at induction modulator as a function of particle energy per nucleon at each momentum error before the correction.

$E_{c}=A e_{c}$ and $E_{d}=A e_{d}$, we normalize the equation as

$$
e_{c}=e_{d}-\frac{\Delta E_{a}}{E_{d}} e_{d}+\frac{q V_{i n d}}{A} \frac{\Delta V_{f}}{V_{i n d}},
$$

where $A$ is the atomic mass number of the beam ion. As written in Eq. (3), the corrected particle energy is restricted by the voltage fluctuation of induction module and the error given at the particle energy analyzer. The corrected momentum error is defined by

$$
\left(\frac{\Delta p}{p}\right)_{c}=\left(\frac{2 m_{u} c^{2} e_{c}+e_{c}^{2}}{2 m_{u} c^{2} e_{d}+e_{d}^{2}}\right)^{\frac{1}{2}}-1,
$$

where $e_{c}=E_{c} / A$ is the corrected particle energy per nucleon.

Using random numbers we calculate the momentum error after the correction. The random number simulates the error at the analyzer and the voltage fluctuation at the induction module. From Eq. (3), we can calculate the corrected particle energy including the detection errors and voltage fluctuations. It is assumed that the random numbers have a normal distribution. Figure 4 shows the rms value of the corrected momentum error versus the analyzing error and voltage fluctuation. When the analyzing error ratio $\Delta E_{a} / E_{d}$ is large $(>0.1 \%)$, the corrected momentum error is independent of the amplitude of the voltage fluctuation. Figure 4 indicates that to get the corrected momentum error less than $10^{-5}$, the voltage fluctuation should be suppressed less than $0.1 \%$, when the analyzed error is less than $10^{-5}$. In practice, the voltage control at fluctuation less than $1 \%$ is difficult under the high voltage pulsed-power operation. The corrected momentum error is substantially limited by the voltage fluctuations at the induction module.

\section{MULTI-TURN OPERATION IN A RING}

Although the solid-state semiconductor devices have good controllability for the trigger timing, the operation at 


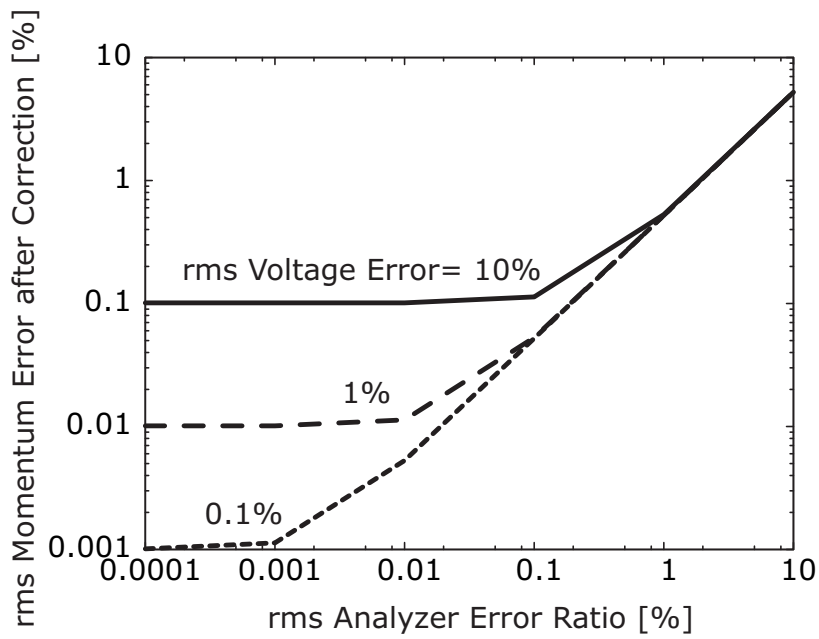

Figure 4: Corrected momentum error as a function of detection error at analyzer and voltage fluctuation of induction modulator. The particle energy per nucleon is assumed as $100 \mathrm{MeV} / \mathrm{u}$. The initial momentum error is $-1 \%$.

the high voltage is difficult to manage and the overlapped voltage is predicted to have a random error. For this reason, the multi-turn voltage additions in a storage ring may be required for the application of this proposed scheme.

Figure 5 shows the typical simulation result for the multi-turn momentum correction. The limitation voltage assumed in the calculation is $100 \mathrm{kV}$, and the controlled voltages are applied to the particle at each lap. The initial momentum error is $-1 \%$ at the central particle energy of $100 \mathrm{MeV} / \mathrm{u}$ with $q / A=0.5$. The coefficient $\left(\Delta V_{f} / V_{\text {ind }}\right)_{0}$ of voltage applied by the induction modulator has the fluctuation of $5 \%$, and the detection error coefficient $\left(\Delta E_{a} / E_{d}\right)_{0}$ at the analyzer of the particle energy is assumed to $10^{-4}$. The ring circumference is $77.76 \mathrm{~m}$, and the revolution period of the beam is $0.6 \mu \mathrm{s}$. As shown in

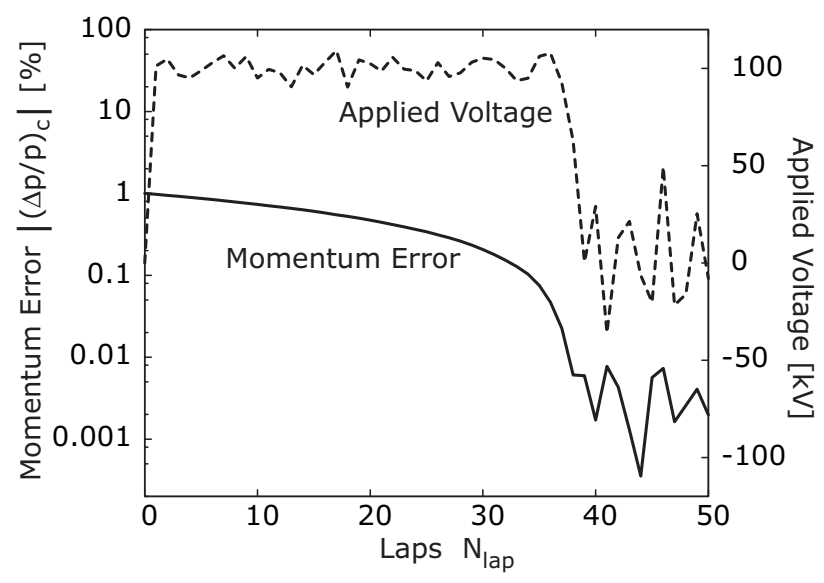

Figure 5: Typical example for momentum error and voltage applied by induction module during multi-laps in a ring.
Fig. 5, the momentum error of the beam rapidly approaches to zero. The rms value of the final momentum error after 50 laps is $5.29 \times 10^{-5}$ for the calculation of 10000 test particles at the total flight time of $30 \mu \mathrm{s}$.

\section{CONCLUSION}

We proposed the selected momentum correction method for single particle with a low ion flux. The error of particle energy detected by the analyzer can be corrected by the voltage applied at the induction modulator. For a single path correction scheme, when the error at the analyzer and voltage fluctuation are small enough, e.g., $\Delta E_{a} / E_{d}=$ $10^{-5}$ and $\Delta V_{f} / V_{\text {ind }}=10^{-3}$, the momentum error of $10^{-5}$ is achieved as shown in Fig. 4. In case of the single path correction scheme, controlling of a high voltage modulator is a challenging problem.

The proposed scenario is much more advantageous with a multi-turn operation in the storage ring, because the achievable final momentum error is smaller than the single path operation with the lower voltage required at the induction module. In this in-situ feedback multi-turn correction scheme, the momentum error rapidly approaches to zero by the correction voltage.

The momentum correction through the single path in an injection line or multi-turn operations in a storage ring may be selected by the situation, such as the initial particle energy, pulse duration, and limitation of maximum voltage applied by the induction module. Since the cooling time of this proposed scheme is very fast, it can be expected as useful tool for experiments with short-lived nuclei.

\section{REFERENCES}

[1] I. Tanihata, H. Hamagaki, O. Hashimoto, Y. Shida, N. Yoshikawa, K. Sugimoto, O. Yamakawa, T. Kobayashi, N. Takahashi, Phys. Rev. Lett. 55, (1985) 2676.

[2] H.-J. Kluge, K. Blaum, C. Scheidenberger, Nucl. Instrum. Methods Phys. Res. A 532, (2004) 48.

[3] B. Franzke, P. Beller, K. Beckert, A. Dolinskii, P. Hulsmann, F. Nolden, C. Peschke, M. Steck, Nucl. Instrum. Methods Phys. Res. A 532, (2004) 97.

[4] Y. Yano, A. Goto, M. Kase, T. Katayama, Proceedings of the 2001 Particle Accelerator Conference, Chicago, (IEEE, Piscataway, NJ, 2001), p.575.

[5] T. Kubo, Nucl. Instrum. Methods Phys. Res. B 204, (2003) 97.

[6] I. Meshkov, W. Mitting, P. Roussel-Chomaz, A. Sidorin, A. Smirnov, E. Syresin, Nucl. Instrum. Methods Phys. Res. A 523, (2004) 262.

[7] K. Horioka, M. Nakajima, M. Watanabe, M. Honda, E. Hotta, M. Shiho, M. Ogawa, J. Hasegawa, J. Kishiro, K. Takayama, Laser Part. Beams 20, (2002) 609.

[8] K. Takayama, et al., Phys. Rev. Lett. 94, (2005) 144801.

[9] H. Kumagai, A. Ozawa, N. Fukuda, K. Summerer, I. Tanihata, Nucl. Instrum. Methods Phys. Res. A 470, (2001) 562. 